\title{
Voluntarily controlled but not merely observed visual feedback affects postural sway
}

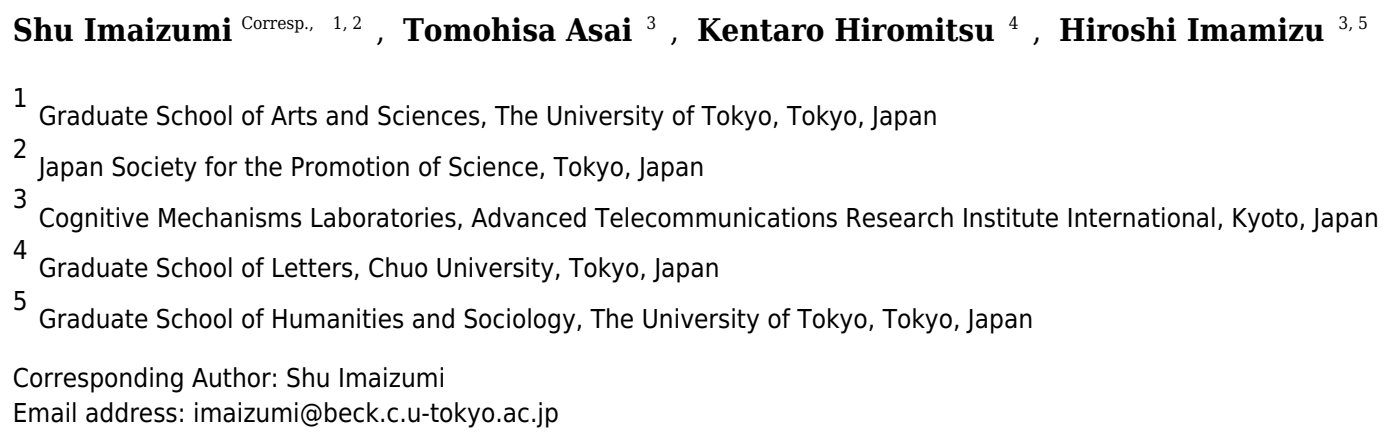

Online stabilization of human standing posture utilizes multisensory afferences (e.g., vision). Whereas visual feedback of spontaneous postural sway can stabilize postural control especially when observers concentrate on their body and intend to minimize postural sway, the effect of intentional control of visual feedback on postural sway itself remains unclear. This study assessed quiet standing posture in healthy adults voluntarily controlling or merely observing visual feedback. The visual feedback (moving square) had either low or high gain and was either horizontally flipped or not. Participants in the voluntary-control group were instructed to minimize their postural sway while voluntarily controlling visual feedback, whereas those in the observation group were instructed to minimize their postural sway while merely observing visual feedback. As a result, magnified and flipped visual feedback increased postural sway only in the voluntarycontrol group. Furthermore, regardless of the instructions and feedback manipulations, the experienced sense of control over visual feedback positively correlated with the magnitude of postural sway. We suggest that voluntarily controlled, but not merely observed, visual feedback is incorporated into the feedback control system for posture and begins to affect postural sway. 
1 Voluntarily controlled but not merely observed visual feedback affects

2

3

4

5

6 Shu Imaizumi ${ }^{1,2}$, Tomohisa Asai ${ }^{3}$, Kentaro Hiromitsu ${ }^{4}$, Hiroshi Imamizu ${ }^{3,5}$

7

8 1. Graduate School of Arts and Sciences, The University of Tokyo, Tokyo, Japan

9 2. Japan Society for the Promotion of Science, Tokyo, Japan

10 3. Cognitive Mechanisms Laboratories, Advanced Telecommunications Research Institute 11 International, Kyoto, Japan

12 4. Graduate School of Letters, Chuo University, Tokyo, Japan

\section{postural sway}

Short title: Voluntary control of postural feedback

5. Graduate School of Humanities and Sociology, The University of Tokyo, Tokyo, Japan

Corresponding author: Shu Imaizumi, imaizumi@beck.c.u-tokyo.ac.jp 


\section{Abstract}

18 Online stabilization of human standing posture utilizes multisensory afferences (e.g., vision).

19 Whereas visual feedback of spontaneous postural sway can stabilize postural control especially

20 when observers concentrate on their body and intend to minimize postural sway, the effect of

21 intentional control of visual feedback on postural sway itself remains unclear. This study

22 assessed quiet standing posture in healthy adults voluntarily controlling or merely observing

23 visual feedback. The visual feedback (moving square) had either low or high gain and was either

24 horizontally flipped or not. Participants in the voluntary-control group were instructed to

25 minimize their postural sway while voluntarily controlling visual feedback, whereas those in the

26 observation group were instructed to minimize their postural sway while merely observing visual

27 feedback. As a result, magnified and flipped visual feedback increased postural sway only in the

28 voluntary-control group. Furthermore, regardless of the instructions and feedback manipulations,

29 the experienced sense of control over visual feedback positively correlated with the magnitude of

30 postural sway. We suggest that voluntarily controlled, but not merely observed, visual feedback

31 is incorporated into the feedback control system for posture and begins to affect postural sway. 


\section{INTRODUCTION}

Human body posture is stabilized by the feedforward and feedback control systems. In the feedforward control system, online comparison between predicted and actual body posture is made on the basis of a predictive signal computed by internal models (Fitzpatrick, Burke \& Gandevia, 1996; van der Kooij et al., 1999). In the feedback control system, concurrent multisensory afferences (i.e., visual, vestibular, and proprioceptive domains) are utilized for online maintenance of body part positions and balance (Mergner \& Rosemeier, 1998; Peterka, 2002). Thus, for instance, unstable body posture during quiet standing can be observed in patients with vestibular disorders (Dozza, Chiari \& Horak, 2005; Fregly, 1974) and in healthy individuals with transient proprioceptive deprivation due to ischemia (Diener et al., 1984). Furthermore, deprivation of visual input by closing the eyes robustly perturbs postural control (Edwards, 1946; Lee \& Lishman, 1975; Travis, 1945). These findings suggest that unisensory information is crucial for intact postural control, even though other sensory modalities retain proper information for postural control.

\section{Postural sway modulated by visual feedback}

The biofeedback technique, by which a quietly standing observer is exposed to additional unisensory stimulation interpreted from the online displacement of his or her center of pressure $(\mathrm{CoP})$ on a force plate, has been utilized for training and rehabilitation for postural control (Litvinenkova \& Hlavacka, 1973; Takeya, Sugano \& Ohno, 1976; Zijlstra et al., 2010). For example, the auditory feedback technique, by which medio-lateral (ML) and antero-posterior (AP) displacements of observers' CoP are converted to a continuous tone of varying volume and pitch and delivered to the observers, has been reported to improve postural control in patients with vestibular disorders (Dozza, Chiari \& Horak, 2005; Dozza, Horak \& Chiari, 2007), whereas some studies have demonstrated the effectiveness of tactile feedback on the tongue (Tyler, Danilov \& Bach-y-Rita, 2003; Vuillerme et al., 2007).

The visual feedback technique, by which observers are presented with the online plot of their CoP displacement on a monitor in the coronal plane parallel to the observers' coronal, has been reported to decrease postural sway (Gantchev, Draganova \& Dunev, 1981; Litvinenkova \& Hlavacka, 1973; Rougier, Farenc \& Berger, 2004; van Peppen et al., 2006; Zijlstra et al., 2010). Literature suggests that there is a stabilizing effect of visual feedback on postural control in 
65

66

67

healthy adults, both young and old, and in patients with altered postural stability (Dault et al., 2003; Freitas \& Duarte, 2012). There is still controversy regarding its effectiveness and feasibility for patients after stroke (Geurts et al., 2005; van Peppen et al., 2006). The mechanism of postural stabilization by visual feedback has considered that the visual feedback provides additional visual inputs in order to integrate multisensory information for the purpose of stabilizing body posture during quiet standing. Some studies have demonstrated that magnification of visual feedback gain relative to actual CoP displacement can further help postural control, because when visual feedback gain is magnified, slight $\mathrm{CoP}$ displacements can be easily detected, facilitating the adjustment of postural control (Cawsey et al., 2009; Jehu, Thibault \& Lajoie, 2016; Rougier, Farenc \& Berger, 2004). Another factor of the biofeedback technique, spatiotemporal (in)congruence of visual feedback has also been studied. Visual feedback with a certain amount of delay (i.e., smaller than $900 \mathrm{~ms}$ ) stabilizes postural control (Rougier, 2004), while larger delays can differentially affect low- and high-frequency fluctuations of CoP displacements (van den Heuvel et al., 2009; Yeh et al., 2010). Horizontallybiased visual feedback requires horizontal compensatory postural adjustments, which can result in increased CoP displacements, but these displacements can be adapted after training (Shiller et al., 2017).

\section{Cognitive effects on postural control and their interactions with visual feedback}

Postural control is also influenced by concurrent cognitive activities. Cognitive tasks performed during quiet standing, such as attentional or working memory tasks, affect postural control by reallocating resources for postural control and cognition. However, studies have reported mixed results, showing either increased, decreased, or unchanged postural sway (Fraizer \& Mitra, 2008). Intentional effort to maintain posture has a key role in maintenance of postural control. Instruction to stand still (i.e., intention to minimize postural sway) has been consistently reported to stabilize postural sway, relative to the result of instruction to relax, although outcome postural indices differ among studies (Loram, Kelly \& Lakie, 2001; Mitra \& Fraizer, 2004; Reynolds, 2010; Stoffregen et al., 2006; Ueta et al., 2015; Zok, Mazza \& Cappozzo, 2008). Instruction can even interfere with the effects of visual feedback on postural sway. For instance, healthy individuals using visual feedback have shown decreased postural sway when they are instructed to stand still, but when they are instructed to relax, they show postural sway comparable to that under non-feedback conditions (Loram, Kelly \& Lakie, 2001). This finding suggests that visual 
97 feedback can be effective in maintaining postural control only when observers intend to

However, it remains unclear whether intentional effort to utilize visual feedback to control posture affects postural sway or interacts with the effect of visual feedback itself. Several studies have already suggested that visuomotor coordination during walking (Malone \& Bastian, 2010) and manual tasks (Benson, Anguera \& Seidler, 2011) can be facilitated by instruction regarding explicit strategies for visual feedback. Given that the feedback system for postural control utilizes concurrent multisensory inputs, including vision, for online adjustment of body posture (Mergner \& Rosemeier, 1998; Peterka, 2002), visual feedback might be particularly able to influence postural sway when observers have an explicitly-guided intention to control both their body posture and its visual feedback so as to accomplish a closed visuo-postural loop. In such a situation, consequently, amplitude and orientation of the visual feedback may be likely to modulate postural sway. Thus, it may be hypothesized that postural control with an intention to control visual feedback might be more influenced by visual feedback and its properties, such as feedback gain (Cawsey et al., 2009; Jehu, Thibault \& Lajoie, 2016; Rougier, Farenc \& Berger, 2004) and spatial orientation (Shiller et al., 2017), than it would be without such intention. Hence, there may be an interactive effect of intentional control and feedback manipulation on postural sway. This study sought to test this hypothesis.

\section{The present study}

We examined whether and how intentional control of concurrent visual feedback of participants' postural sway affects their postural sway. In the present experiment, one group of healthy young adults was instructed to minimize postural sway while voluntarily controlling the concurrent visual feedback of their postural sway presented in a head-mounted display. The other group was instead instructed to merely observe the feedback and not intentionally use it for postural control. This experiment employed a between-participants design to avoid potential carry-over effect and demand characteristics associated with the instructions. Moreover, to examine whether and how the instruction for intentional control enhances the effects of visual feedback properties (e.g., amplitude and orientation) on the postural sway, the visual feedback had two levels of gain and was with or without spatial incongruence (i.e., horizontal flip). We hypothesized that, in participants with explicitly-guided intentions to control visual feedback, high feedback gain 
129 would decrease (e.g., Cawsey et al., 2009) and spatial incongruence between visual feedback and

130 CoP displacement would increase (Shiller et al., 2017) their postural sway.

131

132

133

134

135

136

137

138

139

140

141

142

143

144

145

146

147

148

149

150

151

152

153

154

155

156

157

158

159

160

\section{MATERIALS AND METHODS}

\section{Participants}

Twenty Japanese undergraduates aged 18-22 years participated in the present experiment for monetary compensation of 500 Japanese yen (approximately 4.5 US dollars). Their characteristics are summarized in Table 1. Half of the participants were pseudo-randomly assigned to the voluntary-control group, whereas the other half was assigned to the observation group. The two groups were comparable in sex and age. We also controlled their height (Chiari, Rocchi \& Cappello, 2002), weight (Hue et al., 2007), and body mass index (Greve et al., 2007), each of which may affect postural control. All participants reported that they were right-handed without orthopedic conditions or a history of neurological or psychiatric disorders and had normal visual acuity with or without correction by contact lenses. They also had adequate sleep the night before the experiment. Written informed consent was obtained from each participant prior to the experiment. The present study was conducted in accordance with the Declaration of Helsinki and was approved by the local ethical committee of the Graduate School of Arts and Sciences, The University of Tokyo (approval number: 520).

Sample size was determined based on a priori power analysis using $\mathrm{G}^{*}$ Power 3.1.9.3 (Faul et al., 2007) for an analysis of variance (ANOVA) of the within-between factors, because our main interest was the interactive effect of instruction (i.e., voluntary control, mere observation) on feedback manipulation. The power analysis indicated that at least eight participants for each of the two groups were required for a statistical power of .95, assuming a large effect size in ANOVA $(f=.40$ : Cohen, 1988$)$ and Type I error probability of .05 .

\section{Apparatus}

A force plate (Wii Balance Board, Nintendo, Kyoto, Japan) on a rigid and flat surface tracked the displacements of participants' $\mathrm{CoP}$ on the ML and AP axes with a sampling rate of $30 \mathrm{~Hz}$. The CoP displacement data were collected and sent to a computer (R63/PS, Toshiba, Tokyo, Japan) via Bluetooth interface by an in-house custom program written in Hot Soup Processor 3.4 (ONION Software, Japan) using the open-source library WiiMoteLib 1.7 
161 (http://wiimotelib.codeplex.com) running on Windows 7 Professional 64-bit (Microsoft,

162 Redmond, Washington). The Wii Balance Board has been confirmed as a valid and reliable

163 measurement of postural sway (Clark et al., 2010; Clark et al., 2014; Imaizumi, Asai \& Koyama,

164 2016), while limitations of its measurement precision should also be noted (see Limitations).

Visual feedback of CoP displacement, instructions, and questions were presented on a headmounted display weighing $330 \mathrm{~g}$ (HMZ-T2, Sony, Tokyo, Japan), which had an organic lightemitting diode display with a resolution of $1280 \times 720$ pixels and a refresh rate of $60 \mathrm{~Hz}$ (Hummel et al., 2016). We used a head-mounted display in order to control viewing posture and distance, based on recent evidence suggesting that wearing a head-mounted display is unlikely to affect postural sway during quiet standing (Morel et al., 2015; Robert, Ballaz \& Lemay, 2016) and that effects of instruction (Mitra \& Fraizer, 2004) and visual motion perception (Imaizumi et

\section{Stimuli}

Visual feedback of postural sway (i.e., CoP displacement) was displayed as a white square moving on a coronal plane parallel to the participants' coronal plane (Fig. 1). The square, which subtended at $1.0 \times 1.0^{\circ}$ with a luminance of $28.40 \mathrm{~cd} / \mathrm{m}^{2}$, was presented centrally on a homogeneous black screen $\left(0.40 \mathrm{~cd} / \mathrm{m}^{2}\right)$ at the beginning of each trial. The screen subtended at $45.0 \times 24.7^{\circ}$ with the same aspect ratio as surface of the force plate $(432 \times 237 \mathrm{~mm})$. A $1-\mathrm{mm}$ displacement of CoP on the force plate was synchronously transformed into $0.10^{\circ}$ movement of the white square in the low gain condition and into $0.25^{\circ}$ movement in the high gain condition. Anterior, posterior, leftward, and rightward displacements of $\mathrm{CoP}$ were translated into the upward, downward, leftward, and rightward movements of the square, respectively. We added the horizontally flipped condition, in which the leftward and rightward CoP displacements were translated into the rightward and leftward square movements, respectively. This flip was used to vary the effect of visual feedback on postural control (Shiller et al., 2017) and the subjective feeling of control over the moving square (Asai \& Tanno, 2007; Farrer et al., 2008) by inserting spatial incongruence between bodily movement and visual feedback. In sum, there were four conditions of visual feedback: low gain, low gain flipped, high gain, and high gain flipped.

\section{Procedures}


193 The experiment was conducted individually in a quiet, dimly lit room. After the briefing,

194

195

196

197

198

199

200

201

202

203

204

205

206

207

208

209

210

211

212

213

214

215

216

217

218

219

220

\section{1}

222

223

224

participants removed their wrist and hand ornaments and shoes, put on the head-mounted display, and stood still on the horizontal center of the force plate with their hands down at their sides and their heels together at a $30^{\circ}$ angle between the medial sides of their feet (Kapteyn et al., 1983). Participants were asked to look straight ahead during the experiment.

In each trial, participants' CoP displacements were recorded for 31 seconds while being presented as a moving square on the display (i.e., visual feedback). Participants in both groups were instructed to concentrate on their postural sway and minimize it as much as possible (Reynolds, 2010). They were told that the moving square in the head-mounted display reflected their CoP displacement and postural sway. In the voluntary-control group, they were instructed to minimize their postural sway while voluntarily controlling and utilizing the moving square during the trial. In the observation group, they were instructed to minimize their postural sway while merely observing but not intentionally referring to the moving square. These instructions were presented on the display five seconds before each trial began, in order to inform participants of the task requirements and the onset of recording and stimulus presentation, and to prevent potential postural disturbance associated with stimulus onset and delayed stabilization. To check the validity of the instruction, immediately after each recording of postural sway, the display presented the following question: "To what extent did you feel that you were controlling the moving square?" with an 11-point Likert scale ranging from 0 (i.e., "Not at all") to 10 (i.e., “Extremely"). Participants' vocal responses were recorded by the experimenter. This question was adapted from the one used to measure sense of control over an external object (Evans et al., 2015; Kalckert \& Ehrsson, 2012). Previous studies have used a similar index as that used by the present study to assess the sense of control and to reveal effects of between-participant factors (Imaizumi, Asai \& Koyama, 2016; Kokkinara et al., 2016; Peck et al., 2013). Trials under each of four visual feedback conditions were repeated three times in a randomized order, for a total of 12 trials. The inter-trial intervals were 10 seconds each.

\section{Data analysis}

Recorded CoP displacements during the first 1 second of all trials were excluded from analyses in order to eliminate potential outlying postural sway caused by stimulus onset and/or delayed stabilization. The data from the remaining 30 seconds were analyzed (Kapteyn et al., 1983). We 
225 calculated the total path length, ML path length, AP path length, and enveloped area of the CoP

226 displacements. Total path length was calculated as the sum of the Euclidean distances between

227900 successive data points (i.e., sampled at $30 \mathrm{~Hz}$ for 30 seconds). ML and AP path lengths were

228 calculated as the sum of the ML and AP components, respectively, of the Euclidean distances

229 between data points. Enveloped area was defined as the area enclosed by the outermost path of

230 the CoP displacements. Indices of postural sway were computed using R 3.4.2 (R Core Team,

231 2017). We also used the bivrp package 1.0 (Moral, Hinde \& Demetrio, 2016) to compute

232 enveloped area.

233

234

\section{Statistical analysis}

235 For each participant, each of the abovementioned subjective and postural indices was averaged

236 for the three trials under each visual feedback condition. We first inputted the sense of control

237 rating into a $2 \times 2 \times 2$ ANOVA with a between-factor (Instruction: voluntary control or

238 observation) and two within-factors (Gain: low or high feedback gain; Flip: feedback without or

239 with horizontal flip) in order to check the validity of the instruction. Subsequently, to test the

240 effects of the instructed voluntary control of visual feedback on postural sway and the gain and

241 spatial incongruence (i.e., flip) of visual feedback, we performed the same $2 \times 2 \times 2$ ANOVA on

242 the total, ML, and AP path lengths, and enveloped area. As our interests were mainly in the main

243 effects and interactions of Instruction, we performed post-hoc simple main effect analyses only

244 when significant first- and second-order interactive effects of Instruction were found. Effect sizes

245 in ANOVA were reported as generalized eta squared (Olejnik \& Algina, 2003). Finally, to

246 examine the relationship between the sense of control rating and postural sway in an exploratory

247 manner, we computed Pearson's correlation coefficients between these indices from all

248 participants under each of the four visual-feedback conditions (i.e., the degrees of freedom were

249 78). False discovery rate correction was applied for multiple comparisons (Benjamini \&

250 Hochberg, 1995). Significance level was set at $p<.05$. Hypothesis testing was conducted using

251 SPSS 24.0 (IBM Corp., Armonk, New York) and R 3.4.2 (R Core Team, 2017).

252

253

\section{RESULTS}

254 We performed ANOVA with a between-factor (Instruction) and within-factors (Gain, Flip) on

255 the rating of sense of controlling visual feedback and postural measures. Main effects and

256 interactions of these factors on each measure are summarized in Table 2. 


\section{Sense of control rating: manipulation check}

259

260

261

262

263

264

265

266

267

268

269

270

271

272

273

274

275

276

277

278

279

280

281

282

283

284

285

286

287

288

As expected, the voluntary-control group exhibited higher ratings for experienced sense of control over visual feedback than the observation group did, under all conditions (Fig. 2). This result was supported by a significant main effect of Instruction without any interactions; no effects were found for Gain or Flip (Table 2).

\section{Magnitude of postural sway}

\section{Path length}

Results of the path lengths of CoP displacements are displayed in Fig. 3A-C. We found a second-order Instruction $\times$ Gain $\times$ Flip interaction on the total path length, in addition to Gain $\times$ Flip and Instruction $\times$ Gain interactions (Table 2). Simple interaction analysis revealed that a Gain $\times$ Flip interaction was found in the voluntary-control group $\left(F(1,9)=10.77, p=.010, \eta^{2}{ }_{\mathrm{G}}=\right.$ $.018)$ but not in the observation group $\left(F(1,9)=0.81, p=.390, \eta^{2}<.001\right)$. Simple main effect analysis indicated that, in the voluntary-control group, greater total path length was found in the high gain flipped condition than in the low gain flipped and high gain non-flipped conditions $\left(F(1,9)=10.17, p=.011, \eta_{\mathrm{G}}^{2}=.064 ; F(1,9)=9.73, p=.012, \eta_{\mathrm{G}}^{2}=.039\right.$, respectively $)$.

Furthermore, an Instruction $\times$ Gain interaction was found in the flipped condition $(F(1,18)=9.18$, $\left.p=.007, \eta^{2}{ }_{\mathrm{G}}=.019\right)$ but not in the non-flipped condition $\left(F(1,18)=0.02, p=.900, \eta^{2}{ }_{\mathrm{G}}<.001\right)$, resulting in greater total path length under the high gain flipped condition in the voluntarycontrol group than in the observation group $\left(F(1,18)=4.74, p=.043, \eta^{2}=.209\right)$.

A similar trend was observed for ML path length. There was a significant second-order Instruction $\times$ Gain $\times$ Flip interaction on ML path length (Table 2). Although no first-order interactions were observed, we performed an exploratory simple interaction analysis, revealing that a Gain $\times$ Flip interaction was found in the voluntary-control group $(F(1,9)=5.24, p=.048$, $\left.\eta^{2}{ }_{\mathrm{G}}=.008\right)$ but not in the observation group $\left(F(1,9)=3.02, p=.116, \eta^{2}{ }_{\mathrm{G}}=.002\right)$. An analysis of simple main effect indicated that in the voluntary-control group, greater ML path length was found for the high gain flipped condition than for the low gain flipped and the high gain nonflipped conditions $\left(F(1,9)=6.38, p=.033, \eta^{2}{ }_{\mathrm{G}}=.049 ; F(1,9)=13.56, p=.005, \eta^{2}{ }_{\mathrm{G}}=.034\right.$, respectively). Moreover, an Instruction $\times$ Gain interaction was found in the flipped condition $\left(F(1,18)=5.86, p=.026, \eta^{2}{ }_{\mathrm{G}}=.020\right)$ but not in the non-flipped condition $(F(1,18)=0.21, p=$ 
289

290

291

292

293

294

295

296

297

298

299

300

301

302

303

304

305

306

307

308

309

310

311

312

313

314

\section{5}

316

317

318

319

320

$\left..652, \eta_{\mathrm{G}}^{2}=.001\right)$, resulting in greater ML path length under the high gain flipped condition in the voluntary-control group than in the observation group $\left(F(1,18)=7.23, p=.015, \eta^{2}{ }_{\mathrm{G}}=.287\right)$.

As for AP path length, we found a significant Instruction $\times$ Gain $\times$ Flip second-order interaction in addition to Gain $\times$ Flip and Instruction $\times$ Gain interactions (Table 2). A simple Gain $\times$ Flip interaction was found in the voluntary-control group $\left(F(1,9)=12.53, p=.006, \eta^{2}{ }_{\mathrm{G}}=.023\right)$ but not in the observation group $\left(F(1,9)=0.54, p=.481, \eta^{2}{ }_{\mathrm{G}}<.001\right)$. Simple main effect analysis suggested that, in the voluntary-control group, AP path length was greater under the high gain flipped condition than under the low gain flipped and high gain non-flipped conditions $(F(1,9)=$ $17.03, p=.003, \eta^{2}{ }_{\mathrm{G}}=.075 ; F(1,9)=8.15, p=.019, \eta^{2}{ }_{\mathrm{G}}=.033$, respectively), and smaller AP path length was observed for the low gain flipped condition than in the low gain non-flipped condition $\left(F(1,9)=5.62, p=.042, \eta^{2}{ }_{\mathrm{G}}=.015\right)$.

Taken together, increased gain and spatial incongruence (i.e., flip) of the visual feedback lengthened ML and AP components of the CoP displacements only in the voluntary-control group, although the lengthening effect did not appear under some conditions.

\section{Enveloped area}

Results of the enveloped area of CoP displacements are displayed in Fig. 3D. We found no significant first- and second-order interactions (Table 2). However, given trends toward the significance of Instruction $\times$ Flip interaction $(p=.065)$, we performed exploratory simple main effect analyses. As a result, there was a simple main effect of Flip in the voluntary-control group $\left(F(1,9)=13.57, p=.005, \eta^{2}{ }_{\mathrm{G}}=.118\right)$ but not in the observation group $(F(1,9)=1.24, p=.294$, $\left.\eta^{2}{ }_{\mathrm{G}}=.011\right)$. These indicated that horizontal flip of visual feedback, but not feedback gain, increased the enveloped area of postural sway only in the voluntary-control group.

\section{Correlations among subjective and postural measures}

Table 3 displays correlations between ratings of sense of control over visual feedback and magnitude of postural sway from both groups under each of the four feedback conditions. This analysis allowed us to check how these subjective and postural indices were correlated, regardless of experimental manipulations (i.e., instruction, feedback gain and flip). Results showed that sense of control rating positively correlated with total, ML, and AP path lengths. 
321 These results indicate that stronger sense of control over visual feedback is associated with the

322 greater postural sway in path length. The enveloped area did not correlate with any measures.

323

324

325

326

327

328

329

330

331

332

333

334

335

336

337

338

339

340

341

342

343

344

345

346

347

348

349

350

351

352

\section{DISCUSSION}

The present study examined how intention to control visual feedback of postural sway and modification of visual feedback by gain magnification (low or high) and horizontal flip (with or without) have influences on postural sway. The intention to control was properly manipulated: participants in the voluntary-control group, who were instructed to minimize their postural sway while voluntarily controlling visual feedback, indeed rated their experienced sense of control over visual feedback more highly than did those in the observation group, who were instructed to minimize their postural sway while merely observing visual feedback without intentional reference to it for postural control.

\section{Voluntarily controlled, but not merely observed, visual feedback affects postural stability} We found that, as hypothesized, modification of visual feedback affected postural sway in the voluntary-control group and not in the observation group. Specifically, magnified gain and horizontal flip of the feedback increased path length of CoP displacements in ML and AP directions, whereas the enveloped area of postural sway was increased only by horizontal flip (see below for discussion regarding the difference between path length and area). Previous studies have demonstrated an interactive effect of intention to control body posture on the effect of visual feedback, indicating that visual feedback can affect postural stability only when observers are instructed to minimize postural sway (Loram, Kelly \& Lakie, 2001). In contrast, the present results suggested an interactive effect of intention to control visual feedback on the effect of visual feedback itself, indicating that visual feedback can affect postural stability only when observers voluntarily control the visual feedback. In this situation, even artificially-added visual feedback should be incorporated into the sensorimotor loop in the feedback control system for online adjustments of body posture (Mergner \& Rosemeier, 1998; Peterka, 2002). Although many researches have focused on the effects of additional sensory feedback on postural control (van Peppen et al., 2006; Zijlstra et al., 2010), they might have overlooked how sensory feedback is voluntarily controlled and/or utilized by observers.

However, there seem to be two side effects of intentional control of visual feedback. First, the 
353 voluntary-control group appeared to show greater path lengths and enveloped area in all

354 conditions than did the observation group, although a significant main effect of Instruction was

355 observed only for the ML path length. Explicitly-guided intention to minimize postural sway can

356 robustly decrease postural sway more than just an intention to relax can (Loram, Kelly \& Lakie,

357 2001; Mitra \& Fraizer, 2004; Reynolds, 2010; Stoffregen et al., 2006; Ueta et al., 2015; Zok,

358 Mazza \& Cappozzo, 2008). Moreover, giving attentional focus to external objects while

359 intending to minimize postural sway can also stabilize postural control (McNevin \& Wulf, 2002;

360 Wulf et al., 2004). Given that both groups in our experiment were instructed to minimize

361 postural sway, and individuals in the voluntary-control group would have focused their attention

362 on an external object (i.e., visual feedback), it would be plausible that the apparent differences in

363 postural stability between groups resulted from the effect of intention to control the visual

364 feedback per se. Second, contrary to our prediction, high gain feedback increased three types of

365 path lengths (but only under the flipped conditions), while previous studies have suggested that

366 high gain visual feedback decreases postural sway in healthy individuals (Cawsey et al., 2009;

367 Jehu, Thibault \& Lajoie, 2016; Rougier, Farenc \& Berger, 2004). Possible explanations for the

368 above side effects may be that, in order to voluntarily control and minimize the movement of

369 visual feedback, participants were required to constantly adjust body posture against spontaneous

370 and/or purposeful changes in the body reference configuration during quiet standing (Danna-

371 Dos-Santos et al., 2008). Moreover, when feedback gain was magnified, participants had to

372 adjust their body postures to a greater extent. Although there has been a controversy regarding

373 the efficacy of visual feedback training on postural control (Geurts et al., 2005; van Peppen et al.,

374 2006), it might be speculated that the mixed outcomes of visual feedback training could be due

375 to the lack of investigation on the influence of intentional effort to use visual feedback to adjust

376 body posture.

377

378

Although intentional control of visual feedback may cause perturbing side effects, our

379 correlation analysis indicated that there were positive correlations between sense of control

380 ratings and total, ML, and AP path lengths across groups and conditions. This suggests that in order for additional visual feedback to affect postural control, the existence of both instruction to voluntarily control visual feedback and the experienced sense of control over the visual feedback are important, regardless of the magnification and spatial bias of the feedback. We should point out that even though the observation group was instructed not to intend to control the visual 
385 feedback, they did not indicate that they felt no sense of control (mean scores ranging

386 approximately 2.0-3.5, see Fig. 2). It can be speculated that although the observation group did

387 not have a priori intention to control visual feedback, they might have experienced a sense of

388 control unconsciously generated from post-hoc inference (Synofzik, Vosgerau \& Voss, 2013;

389 Wegner, 2003), because they knew that the movement of visual feedback corresponded to their

390 own CoP displacement. If so, in their violation of the instruction provided to the observation

391 group, visual feedback might have had an influence on their postural control.

392

393 We should also clarify that the effects of gain magnification and horizontal flip on path length in

394 the voluntary-control group were apparent only when both of them were applied, suggesting that

395 each of these feedback modifications by itself was not strong enough to demonstrate an effect.

396 While a previous finding suggests that horizontal flip of visual feedback could cause postural

397 instability (Shiller et al., 2017), in our low gain conditions, the flip did not result in any effect on

398 total and ML path lengths, and even had a stabilizing effect on AP path length. This might be

399 because participants may have had difficulty in detecting horizontal flip because of the low

400 degree of feedback gain, and, consequently, the flip did not perturb their postural control. If this

401 is the case, this explanation also accounts for the perturbation effect of horizontal flip in high

402 gain conditions: participants could detect flip because of the large amount of visual feedback

403 movement, and the flip thus affected their postural control. Nevertheless, horizontal flip

404 increased enveloped area, regardless of feedback gain. This may highlight the lack of

405 magnification of visual feedback in the high gain condition, and also suggest a potentially

406 different nature of path length and enveloped area. Regarding sufficient amounts of feedback

407 gain, relative difference between a high gain of $0.25^{\circ}$ and a low gain of $0.10^{\circ}$ corresponding to 1

$408 \mathrm{~mm}$ CoP displacement might not be enough to increase postural sway, given that previous

409 studies have reported that visual feedback with gains of $1.43^{\circ}$ relative to $0.14^{\circ}$ (Rougier, 2005)

410 and $0.29^{\circ}$ relative to $0.06^{\circ}$ (Jehu, Thibault \& Lajoie, 2016) decreased more postural sway (note

411 that the authors transformed the original $\mathrm{cm}$ gain values into those of visual angles based on

412 viewing distances reported in the cited papers). Further studies are needed to elucidate

413 relationship between visual feedback modifications and intentional control of visual feedback, by

414 applying wide-ranged, finely varied feedback gains and spatial rotations.

415

416 Differences between sway path length and area 
417 In our experiment, path lengths and the enveloped area of CoP displacements showed different

418 tendencies in the effect of feedback modifications and different relationships with sense of

419 control. The voluntary-control group exhibited total, ML, and AP path lengths subject to the

420 effects of feedback gain and horizontal flip, and enveloped area affected only by horizontal flip.

421 Furthermore, there was no correlation between path lengths and enveloped area. These results

422 suggest a different nature of these indices, which may be interpreted by their different origins:

423 sway path length, which reflects how frequently CoP fluctuates, originates mainly from the

424 proprioceptive and motor systems (Mauritz \& Dietz, 1980); in contrast, sway area, which reflects

425 how widely CoP fluctuates, can originate from vestibular function (Kapteyn \& de Wit, 1972), in

426 addition to being influenced by the mechanical properties of body posture. We found that the

427 three path lengths and sense of control rating correlated with each other, while the enveloped

428 area did not correlate with any indices. These results not only further suggest the differences

429 between sway path length and area, but may also indicate that sense of control contributes more

430 to the motor-related sway component (i.e., path length) constituting a closed visuo-motor loop.

431

432 Sense of control unaffected by visual feedback modifications

433 The subjective rating of the sense of control over visual feedback of one's own actions has been

434 reported to be affected by intensity and spatial congruence of visual feedback. For example,

435 faster movement of dots triggered by an observer's key press is likely to result in a stronger

436 sense of control over the moving dots (Kawabe, 2013). Moreover, angular biases inserted into

437 visual feedback of observers' manual actions using a joystick and computer mouse can reduce

438 sense of control over the visual feedback (Asai \& Tanno, 2007; Farrer et al., 2008). Contrary to

439 our prediction made from these previous findings, the present results showed that sense of

440 control over visual feedback of postural sway was not affected by feedback gain and spatial

441 incongruence (i.e., flip). There were two potential explanations. First, the quantity of gain

442 magnification was not enough to increase sense of control. Indeed, Kawabe (2013) reported that

$4438.5 \%$ sec movement of dots initiated by participants' key press induced stronger sense of control

444 than $\operatorname{did} 2.1 \%$ sec movement, while $4.2^{\circ} / \mathrm{sec}$ movement did not induce stronger sense of control

445 than the $2.1 / \mathrm{sec}$ movement. Thus, our "high" feedback gain might indeed not be high enough to

446 increase sense of control.

447

448 A second potential explanation, although speculative, is that the null effect on sense of control 
449 rating might be because of a potential difference between visuo-manual and visuo-postural

450 relationships. Sense of control over external objects and sense of agency over one's own actions

451 have been thought to stem from an internal forward model of the sensorimotor system in the

452 brain (Frith, Blakemore \& Wolpert, 2000), which includes the predictor and its comparator in

453 order to match predicted and actual sensory feedbacks based on motor commands (Wolpert,

454 Ghahramani \& Jordan, 1995). Although many studies have experimentally manipulated

455 spatiotemporal (in)congruence between sensory feedback and manual action and revealed the

456 mechanisms of senses of control and agency (David, Newen \& Vogeley, 2008; Haggard, 2017),

457 little is known about the sense of control over sensory feedback of full-body movement such as

458 postural control, except for locomotion (Kannape \& Blanke, 2013; Kannape et al., 2010). Given

459 that body posture is stabilized based not only on the predictive feedforward control system

460 (Fitzpatrick, Burke \& Gandevia, 1996), but also on the responsive feedback control system

461 (Peterka, 2002), sense of control over sensory feedback of postural sway may arise in a manner

462 different from that of manual action, whereby sense of control in postural control weighs its

463 dependence less on the internal forward model than it does in manual action. Alternatively, we

464 might assume that if the forward model is unlikely to predict single visual event (e.g., moving

465 square) in the ecological environments as a consequence of postural sway and/or full-body

466 movement, other than optic flow (Fajen, 2007), sense of control would not be affected by

467 (in)congruence of visual feedback, regardless of gain and flip.

468

469 Limitations

470 We have three limitations of note. First, although validity of the Wii Balance Board for postural

471 measurement has been confirmed (e.g., Clark et al., 2010), recent studies have suggested its

472 limited measurement precision, such as inconsistent sampling rate and poor signal to noise ratio

473 (Clark et al., 2018; Leach et al., 2014). Our results might have been affected because we sampled

474 the data using a constant rate within our custom program but did not calibrate the force

475 parameter from the Wii Balance Board. Second, because we gave instructions to manipulate

476 participants' intention to control the visual feedback of postural sway and assessed the

477 experienced sense of control by subjective rating, we cannot exclude a possibility that the sense

478 of control rating might have been biased by demand characteristics. Considering that participants'

479 responses did not approach extreme values and varied across individuals (i.e., the voluntary-

480 control group scored approximately 4-6, while the observation group scored approximately 2-4, 
481 Fig. 2), it may be plausible that the group difference in the responses were not biased and caused

482 by the instructions. Nevertheless, to address this potential issue, objective measures could be

483 helpful. For example, intention to control and/or experienced sense of control may be reflected in

484 the time-series regularity of postural fluctuations, such as fractality and self-affinity (Delignieres

485 et al., 2003), as the degree of cognitive involvement in postural control (e.g., attention) can

486 indeed be reflected in the postural time-series regularities (Donker et al., 2007; Stins et al., 2009).

487 Finally, we employed instructions to and not to intentionally control visual feedback as a

488 between-participants factor, in order to avoid potential carry-over effects and demand

489 characteristics, which might affect the results. However, we cannot exclude the potential

490 confounding effects of any individual differences in postural control and susceptibility to

491 instruction. Further investigation to overcome the above limitations would facilitate deeper

492 understanding of the effect of voluntary control over visual feedback on postural control.

493

494

495

CONCLUSIONS

496 of postural sway in a quiet standing position can affect postural sway, but only when individuals

497 intend to control the movement of visual feedback; moreover, the experienced sense of control

498 correlates with postural sway. Voluntarily controlled, but not merely observed, visual feedback

499 may be incorporated into the feedback postural-control system and affect postural sway.

501 Our findings contribute to an increased understanding of the potential role of intention and

502 mental set in postural biofeedback techniques for healthy and impaired individuals. Although

503 speculative, mixed outcomes from trials using postural biofeedback (Geurts et al., 2005; van

504 Peppen et al., 2006) might have resulted from a lack of consideration for the role of intentional

505 control over the biofeedback. Thus, one direction for future clinical and therapeutic studies may

506 include development of a new training protocol. It may be fruitful to further investigate how

507 intentional control of sensory feedback and an experienced sense of control can influence

508 postural control in patients who have experienced a stroke (Shumway-Cook, Anson \& Haller,

509 1988), have a vestibular disorder (Fregly, 1974), or whose postural stability is likely to be

510 perturbed. An additional future direction, from the perspective of psychology and psychiatry,

511 would be to elucidate the relationships between sense of control or agency and whole-body

512 movement (i.e., postural control and gait), particularly in schizophrenic and schizotypal 
513 individuals, who tend to experience a weakened sense of control and impaired self-other

514 discrimination (Asai, 2016; Franck et al., 2001), in addition to healthy individuals.

515

516

517

518

519

520

521

522

523

524

525

526

527

528

529

530

531

532

533

534

535

536

537

538

539

540

541

542

543

544

\section{ACKNOWLEDGMENTS}

We would like to thank Ryota Hatakama for his help during data collection and three reviewers for their helpful comments on a previous version of the manuscript. The pilot experiment for this study was conducted in the NTT Communication Science Laboratories where SI and TA had formerly worked.

Asai T. 2016. Self is "other", other is "self": poor self-other discriminability explains schizotypal twisted agency judgment. Psychiatry Research 246:593-600. DOI 10.1016/j.psychres.2016.10.082

Asai T, Tanno Y. 2007. The relationship between the sense of self-agency and schizotypal personality traits. Journal of Motor Behavior 39:162-168. DOI 10.3200/JMBR.39.3.162-168

Benjamini Y, Hochberg Y. 1995. Controlling the false discovery rate: a practical and powerful approach to multiple testing. Journal of the Royal Statistical Society: Series B 57:289-300. DOI 10.2307/2346101

Benson BL, Anguera JA, Seidler RD. 2011. A spatial explicit strategy reduces error but interferes with sensorimotor adaptation. Journal of Neurophysiology 105:2843-2851. DOI 10.1152/jn.00002.2011

Cawsey RP, Chua R, Carpenter MG, Sanderson DJ. 2009. To what extent can increasing the magnification of visual feedback of the centre of pressure position change the control of quiet standing balance? Gait and Posture 29:280-284. DOI 10.1016/j.gaitpost.2008.09.007

Chiari L, Rocchi L, Cappello A. 2002. Stabilometric parameters are affected by anthropometry and foot placement. Clinical Biomechanics 17:666-677. DOI 10.1016/S02680033(02)00107-9

Clark RA, Bryant AL, Pua Y, McCrory P, Bennell K, Hunt M. 2010. Validity and reliability of the Nintendo Wii Balance Board for assessment of standing balance. Gait and Posture 31:307-310. DOI 10.1016/j.gaitpost.2009.11.012

Clark RA, Hunt M, Bryant AL, Pua Y. 2014. Author response to the letter: on "Validity and reliability of the Nintendo Wii Balance Board for assessment of standing balance": are the 
545

546

547

548

549

550

551

552

553

554

555

556

557

558

559

560

561

562

563

564

565

566

567

568

569

570

571

572

573

574

575

576

conclusions stated by the authors justified? Gait and Posture 39:1151-1154. DOI 10.1016/j.gaitpost.2013.12.013

Clark RA, Mentiplay BF, Pua YH, Bower KJ. 2018. Reliability and validity of the Wii Balance Board for assessment of standing balance: A systematic review. Gait and Posture 61:40-54. DOI 10.1016/j.gaitpost.2017.12.022

Cohen J. 1988. Statistical Power Analysis for the Behavioral Sciences. Mahwah, New Jersey: Lawrence Erlbaum Associates.

Danna-Dos-Santos A, Degani AM, Zatsiorsky VM, Latash ML. 2008. Is voluntary control of natural postural sway possible? Journal of Motor Behavior 40:179-185. DOI 10.3200/JMBR.40.3.179-185

Dault MC, de Haart M, Geurts AC, Arts IM, Nienhuis B. 2003. Effects of visual center of pressure feedback on postural control in young and elderly healthy adults and in stroke patients. Human Movement Science 22:221-236. DOI 10.1016/S0167-9457(03)00034-4

David N, Newen A, Vogeley K. 2008. The "sense of agency" and its underlying cognitive and neural mechanisms. Consciousness and Cognition 17:523-534. DOI

10.1016/j.concog.2008.03.004

Delignieres D, Deschamps T, Legros A, Caillou N. 2003. A methodological note on nonlinear time series analysis: is the open- and closed-loop model of Collins and De Luca (1993) a statistical artifact? Journal of Motor Behavior 35:86-97. DOI 10.1080/00222890309602124

Diener HC, Dichgans J, Guschlbauer B, Mau H. 1984. The significance of proprioception on postural stabilization as assessed by ischemia. Brain Research 296:103-109. DOI

10.1016/0006-8993(84)90515-8

Donker SF, Roerdink M, Greven AJ, Beek PJ. 2007. Regularity of center-of-pressure trajectories depends on the amount of attention invested in postural control. Experimental Brain Research 181:1-11. DOI 10.1007/s00221-007-0905-4

Dozza M, Chiari L, Horak FB. 2005. Audio-biofeedback improves balance in patients with bilateral vestibular loss. Archives of Physical Medicine and Rehabilitation 86:1401-1403. DOI 10.1016/j.apmr.2004.12.036

Dozza M, Horak FB, Chiari L. 2007. Auditory biofeedback substitutes for loss of sensory information in maintaining stance. Experimental Brain Research 178:37-48. DOI 10.1007/s00221-006-0709-y

Edwards AS. 1946. Body sway and vision. Journal of Experimental Psychology 36:526-535. 
577

578

579

580

581

582

583

584

585

586

587

588

589

590

591

592

593

594

595

596

597

598

599

600

601

602

603

604

605

606

607

608

\section{DOI $10.1037 / \mathrm{h} 0059909$}

Evans N, Gale S, Schurger A, Blanke O. 2015. Visual feedback dominates the sense of agency for brain-machine actions. PLOS ONE 10:e0130019. DOI 10.1371/journal.pone.0130019

Fajen BR. 2007. Rapid recalibration based on optic flow in visually guided action. Experimental Brain Research 183:61-74. DOI 10.1007/s00221-007-1021-1

Farrer C, Bouchereau M, Jeannerod M, Franck N. 2008. Effect of distorted visual feedback on the sense of agency. Behavioural Neurology 19:53-57. DOI 10.1155/2008/425267

Faul F, Erdfelder E, Lang AG, Buchner A. 2007. G*Power 3: a flexible statistical power analysis program for the social, behavioral, and biomedical sciences. Behavior Research Methods

$$
\text { 39:175-191. DOI 10.3758/bf03193146 }
$$

Fitzpatrick R, Burke D, Gandevia SC. 1996. Loop gain of reflexes controlling human standing measured with the use of postural and vestibular disturbances. Journal of Neurophysiology 76:3994-4008. DOI 10.1152/jn.1996.76.6.3994

Fraizer EV, Mitra S. 2008. Methodological and interpretive issues in posture-cognition dualtasking in upright stance. Gait and Posture 27:271-279. DOI 10.1016/j.gaitpost.2007.04.002

Franck N, Farrer C, Georgieff N, Marie-Cardine M, Dalery J, d'Amato T, Jeannerod M. 2001. Defective recognition of one's own actions in patients with schizophrenia. American Journal of Psychiatry 158:454-459. DOI 10.1176/appi.ajp.158.3.454

Fregly AR. 1974. Vestibular ataxia and its measurement in man. In: Kornhuber HH, ed. Vestibular System Part 2: Psychophysics, Applied Aspects and General Interpretations. Berlin: Springer Berlin Heidelberg, 321-360.

Freitas SM, Duarte M. 2012. Joint coordination in young and older adults during quiet stance: effect of visual feedback of the center of pressure. Gait and Posture 35:83-87. DOI 10.1016/j.gaitpost.2011.08.011

Frith CD, Blakemore SJ, Wolpert DM. 2000. Abnormalities in the awareness and control of action. Philosophical Transactions of the Royal Society of London Series B: Biological Sciences 355:1771-1788. DOI 10.1098/rstb.2000.0734

Gantchev G, Draganova N, Dunev S. 1981. Role of the visual feedback in postural control. Agressologie 22:59-62.

Geurts AC, de Haart M, van Nes IJ, Duysens J. 2005. A review of standing balance recovery from stroke. Gait and Posture 22:267-281. DOI 10.1016/j.gaitpost.2004.10.002

Greve J, Alonso A, Bordini ACPG, Camanho GL. 2007. Correlation between body mass index 
609

610

611

612

613

614

615

616

617

618

619

620

621

622

623

624

625

626

627

628

629

630

631

632

633

634

635

636

637

638

639

640

and postural balance. Clinics 62:717-720. DOI 10.1590/s1807-59322007000600010

Haggard P. 2017. Sense of agency in the human brain. Nature Reviews: Neuroscience 18:196207. DOI 10.1038/nrn.2017.14

Hue O, Simoneau M, Marcotte J, Berrigan F, Dore J, Marceau P, Marceau S, Tremblay A, Teasdale N. 2007. Body weight is a strong predictor of postural stability. Gait and Posture 26:32-38. DOI 10.1016/j.gaitpost.2006.07.005

Hummel N, Cuturi LF, MacNeilage PR, Flanagin VL. 2016. The effect of supine body position on human heading perception. Journal of Vision 16:19. DOI 10.1167/16.3.19

Imaizumi S, Asai T, Koyama S. 2016. Embodied prosthetic arm stabilizes body posture, while unembodied one perturbs it. Consciousness and Cognition 45:75-88. DOI 10.1016/j.concog.2016.08.019

Imaizumi S, Honma M, Hibino H, Koyama S. 2015. Illusory visual motion stimulus elicits postural sway in migraine patients. Frontiers in Psychology 6:542. DOI 10.3389/fpsyg.2015.00542

Jehu DA, Thibault J, Lajoie Y. 2016. Magnifying the scale of visual biofeedback improves posture. Applied Psychophysiology and Biofeedback 41:151-155. DOI 10.1007/s10484-0159324-7

Kalckert A, Ehrsson HH. 2012. Moving a rubber hand that feels like your own: a dissociation of ownership and agency. Frontiers in Human Neuroscience 6:40. DOI 10.3389/fnhum.2012.00040

Kannape OA, Blanke O. 2013. Self in motion: sensorimotor and cognitive mechanisms in gait agency. Journal of Neurophysiology 110:1837-1847. DOI 10.1152/jn.01042.2012

Kannape OA, Schwabe L, Tadi T, Blanke O. 2010. The limits of agency in walking humans. Neuropsychologia 48:1628-1636. DOI 10.1016/j.neuropsychologia.2010.02.005

Kapteyn TS, Bles W, Njiokiktjien CJ, Kodde L, Massen CH, Mol JM. 1983. Standardization in platform stabilometry being a part of posturography. Agressologie 24:321-326.

Kapteyn TS, de Wit G. 1972. Posturography as an auxiliary in vestibular investigation. Acta OtoLaryngologica 73:104-111. DOI 10.3109/00016487209138918

Kawabe T. 2013. Inferring sense of agency from the quantitative aspect of action outcome. Consciousness and Cognition 22:407-412. DOI 10.1016/j.concog.2013.01.006

Kokkinara E, Kilteni K, Blom KJ, Slater M. 2016. First Person Perspective of Seated Participants Over a Walking Virtual Body Leads to Illusory Agency Over the Walking. 
641

642

643

644

645

646

647

648

649

650

651

652

653

654

655

656

657

658

659

660

661

662

663

664

665

666

667

668

669

670

671

672

Scientific Reports 6:28879. DOI 10.1038/srep28879

Leach JM, Mancini M, Peterka RJ, Hayes TL, Horak FB. 2014. Validating and calibrating the Nintendo Wii balance board to derive reliable center of pressure measures. Sensors 14:18244-18267. DOI 10.3390/s141018244

Lee DN, Lishman JR. 1975. Visual proprioceptive control of stance. Journal of Human Movement Studies 1:87-95.

Litvinenkova V, Hlavacka F. 1973. The visual feed-back gain influence upon the regulation of the upright posture in man. Agressologie 14:95-99.

Loram ID, Kelly SM, Lakie M. 2001. Human balancing of an inverted pendulum: is sway size controlled by ankle impedance? The Journal of Physiology 532:879-891. DOI 10.1111/j.1469-7793.2001.0879e.x

Malone LA, Bastian AJ. 2010. Thinking about walking: effects of conscious correction versus distraction on locomotor adaptation. Journal of Neurophysiology 103:1954-1962. DOI 10.1152/jn.00832.2009

Mauritz KH, Dietz V. 1980. Characteristics of postural instability induced by ischemic blocking of leg afferents. Experimental Brain Research 38:117-119. DOI 10.1007/bf00237939

McNevin NH, Wulf G. 2002. Attentional focus on supra-postural tasks affects postural control. Human Movement Science 21:187-202. DOI 10.1016/S0167-9457(02)00095-7

Mergner T, Rosemeier T. 1998. Interaction of vestibular, somatosensory and visual signals for postural control and motion perception under terrestrial and microgravity conditions: a conceptual model. Brain Research Reviews 28:118-135. DOI 10.1016/S01650173(98)00032-0

Mitra S, Fraizer EV. 2004. Effects of explicit sway-minimization on postural-suprapostural dualtask performance. Human Movement Science 23:1-20. DOI 10.1016/j.humov.2004.03.003

Moral RA, Hinde J, Demetrio CGB. 2016. bivrp: bivariate residual plots with simulation polygons. R package version 1.0.

Morel M, Bideau B, Lardy J, Kulpa R. 2015. Advantages and limitations of virtual reality for balance assessment and rehabilitation. Neurophysiologie Clinique 45:315-326. DOI 10.1016/j.neucli.2015.09.007

Olejnik S, Algina J. 2003. Generalized eta and omega squared statistics: measures of effect size for some common research designs. Psychological Methods 8:434-447. DOI 10.1037/1082989X.8.4.434 
673 Peck TC, Seinfeld S, Aglioti SM, Slater M. 2013. Putting yourself in the skin of a black avatar

674

675

676

677

678

679

680

681

682

683

684

685

686

687

688

689

690

691

692

693

694

695

696

697

698

699

700

701

702

703

704

\section{reduces implicit racial bias. Consciousness and Cognition 22:779-787. DOI}

10.1016/j.concog.2013.04.016

Peterka RJ. 2002. Sensorimotor integration in human postural control. Journal of Neurophysiology 88:1097-1118. DOI 10.1152/jn.2002.88.3.1097

R Core Team. 2017. R: A language and environment for statistical computing. Vienna, Austria: R Foundation for Statistical Computing.

Reynolds RF. 2010. The ability to voluntarily control sway reflects the difficulty of the standing task. Gait and Posture 31:78-81. DOI 10.1016/j.gaitpost.2009.09.001

Robert MT, Ballaz L, Lemay M. 2016. The effect of viewing a virtual environment through a head-mounted display on balance. Gait and Posture 48:261-266. DOI 10.1016/j.gaitpost.2016.06.010

Rougier P. 2004. Optimising the visual feedback technique for improving upright stance maintenance by delaying its display: behavioural effects on healthy adults. Gait and Posture 19:154-163. DOI 10.1016/S0966-6362(03)00056-0

Rougier P. 2005. Compatibility of postural behavior induced by two aspects of visual feedback: time delay and scale display. Experimental Brain Research 165:193-202. DOI $10.1007 / \mathrm{s} 00221-005-2288-8$

Rougier P, Farenc I, Berger L. 2004. Modifying the gain of the visual feedback affects undisturbed upright stance control. Clinical Biomechanics 19:858-867. DOI 10.1016/j.clinbiomech.2004.04.013

Shiller DM, Veilleux LN, Marois M, Ballaz L, Lemay M. 2017. Sensorimotor adaptation of whole-body postural control. Neuroscience 356:217-228. DOI 10.1016/j.neuroscience.2017.05.029

Shumway-Cook A, Anson D, Haller S. 1988. Postural sway biofeedback: its effect on reestablishing stance stability in hemiplegic patients. Archives of Physical Medicine and Rehabilitation 69:395-400.

Stins JF, Michielsen ME, Roerdink M, Beek PJ. 2009. Sway regularity reflects attentional involvement in postural control: effects of expertise, vision and cognition. Gait and Posture 30:106-109. DOI 10.1016/j.gaitpost.2009.04.001

Stoffregen TA, Hove P, Schmit J, Bardy BG. 2006. Voluntary and involuntary postural responses to imposed optic flow. Motor Control 10:24-33. DOI 10.1123/mcj.10.1.24 
705 Synofzik M, Vosgerau G, Voss M. 2013. The experience of agency: an interplay between

706 prediction and postdiction. Frontiers in Psychology 4:127. DOI 10.3389/fpsyg.2013.00127

707 Takeya T, Sugano H, Ohno Y. 1976. Auditory and visual feedback of postural sway.

$708 \quad$ Agressologie 17:71-74.

709 Travis RC. 1945. An experimental analysis of dynamic and static equilibrium. Journal of

$710 \quad$ Experimental Psychology 35:216-234. DOI 10.1037/h0059788

711 Tyler M, Danilov Y, Bach-y-Rita P. 2003. Closing an open-loop control system: vestibular

712 substitution through the tongue. Journal of Integrative Neuroscience 2:159-164. DOI

$713 \quad 10.1142 / \mathrm{s} 0219635203000263$

714 Ueta K, Okada Y, Nakano H, Osumi M, Morioka S. 2015. Effects of voluntary and automatic

715 control of center of pressure sway during quiet standing. Journal of Motor Behavior 47:256-

716 264. DOI 10.1080/00222895.2014.974496

717 van den Heuvel MR, Balasubramaniam R, Daffertshofer A, Longtin A, Beek PJ. 2009. Delayed

718 visual feedback reveals distinct time scales in balance control. Neuroscience Letters 452:37-

719 41. DOI 10.1016/j.neulet.2009.01.024

720 van der Kooij H, Jacobs R, Koopman B, Grootenboer H. 1999. A multisensory integration model

721 of human stance control. Biological Cybernetics 80:299-308. DOI 10.1007/s004220050527

722 van Peppen RP, Kortsmit M, Lindeman E, Kwakkel G. 2006. Effects of visual feedback therapy

723 on postural control in bilateral standing after stroke: a systematic review. Journal of

724 Rehabilitation Medicine 38:3-9. DOI 10.1080/16501970500344902

725 Vuillerme N, Chenu O, Demongeot J, Payan Y. 2007. Controlling posture using a plantar

726 pressure-based, tongue-placed tactile biofeedback system. Experimental Brain Research

727 179:409-414. DOI 10.1007/s00221-006-0800-4

728 Wegner DM. 2003. The mind's best trick: how we experience conscious will. Trends in

729 Cognitive Sciences 7:65-69. DOI 10.1016/s1364-6613(03)00002-0

730 Wolpert DM, Ghahramani Z, Jordan MI. 1995. An internal model for sensorimotor integration.

731 Science 269:1880-1882. DOI 10.1126/science.7569931

732 Wulf G, Mercer J, McNevin N, Guadagnoli MA. 2004. Reciprocal influences of attentional focus

733 on postural and suprapostural task performance. Journal of Motor Behavior 36:189-199.

734 DOI 10.3200/JMBR.36.2.189-199

735 Yeh TT, Boulet J, Cluff T, Balasubramaniam R. 2010. Contributions of delayed visual feedback

736 and cognitive task load to postural dynamics. Neuroscience Letters 481:173-177. DOI 
$737 \quad$ 10.1016/j.neulet.2010.06.081

738 Zijlstra A, Mancini M, Chiari L, Zijlstra W. 2010. Biofeedback for training balance and mobility 739 tasks in older populations: a systematic review. Journal of Neuroengineering and

$740 \quad$ Rehabilitation 7:58. DOI 10.1186/1743-0003-7-58

741 Zok M, Mazza C, Cappozzo A. 2008. Should the instructions issued to the subject in traditional 742 static posturography be standardised? Medical Engineering and Physics 30:913-916. DOI

$743 \quad$ 10.1016/j.medengphy.2007.12.002 
Figure 1 (on next page)

Schematic of the visual feedback of postural sway.

(A) The force plate tracked the displacement of participants' center of pressure. (B) The center of pressure displacement of $1 \mathrm{~mm}$ on the force plate corresponded to 0.10 and $0.25^{\circ}$ displacement of a white square on the black screen in head-mounted display under the low and high gain conditions, respectively. For example, in the non-flipped and flipped conditions, when the center of pressure moved to the front left, the white square moved to the upper left and right, respectively. 
A

B

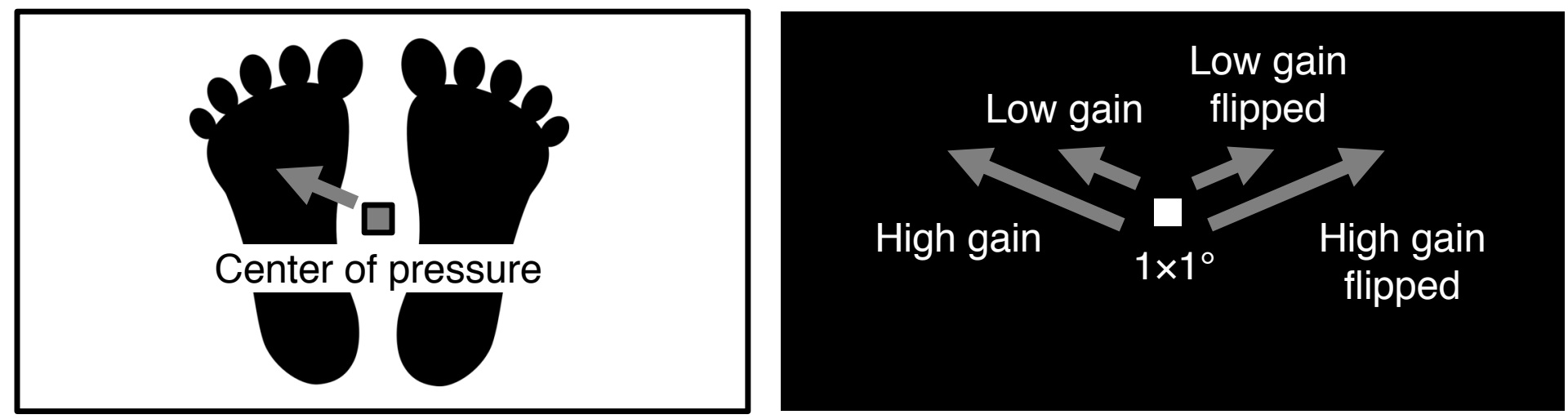




\section{Figure 2 (on next page)}

Subjective rating of the sense of control over visual feedback of postural sway.

Error bars denote standard error of the mean. Asterisks indicate a significant difference between groups $\left({ }^{* * *} p<.001\right)$. 


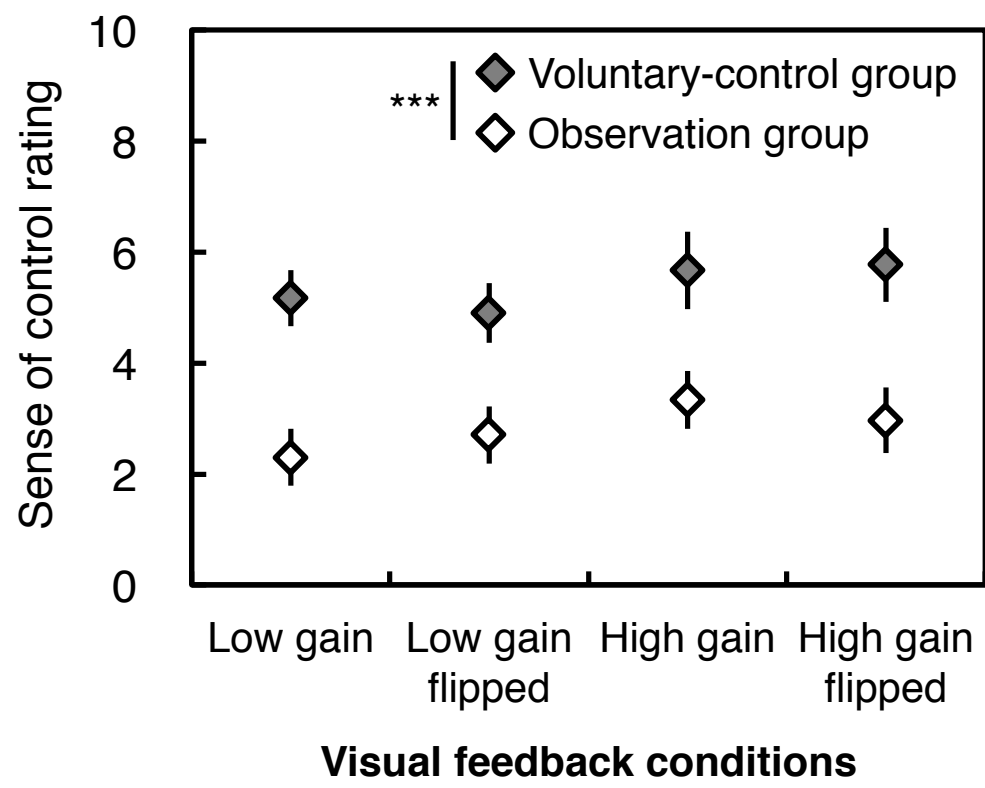




\section{Figure 3 (on next page)}

Magnitude of postural sway.

(A) Total path length, (B) medio-lateral (ML) path length, (C) antero-posterior (AP) path length, and (D) enveloped area of the center of pressure displacements. Error bars denote standard error of the mean. Asterisks indicate significant simple main effects $\left({ }^{*} p<.05,{ }^{* *} p<\right.$ $.01)$. 
A

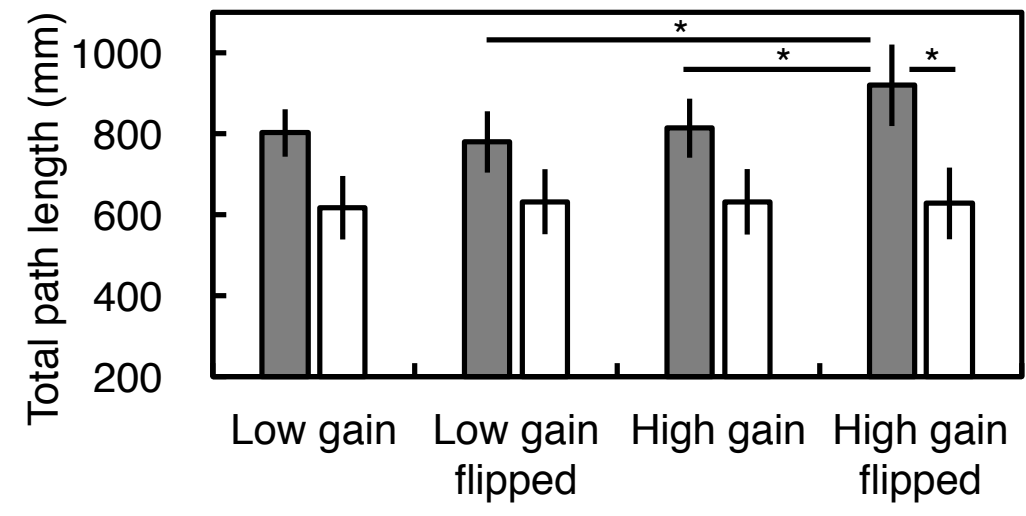

C

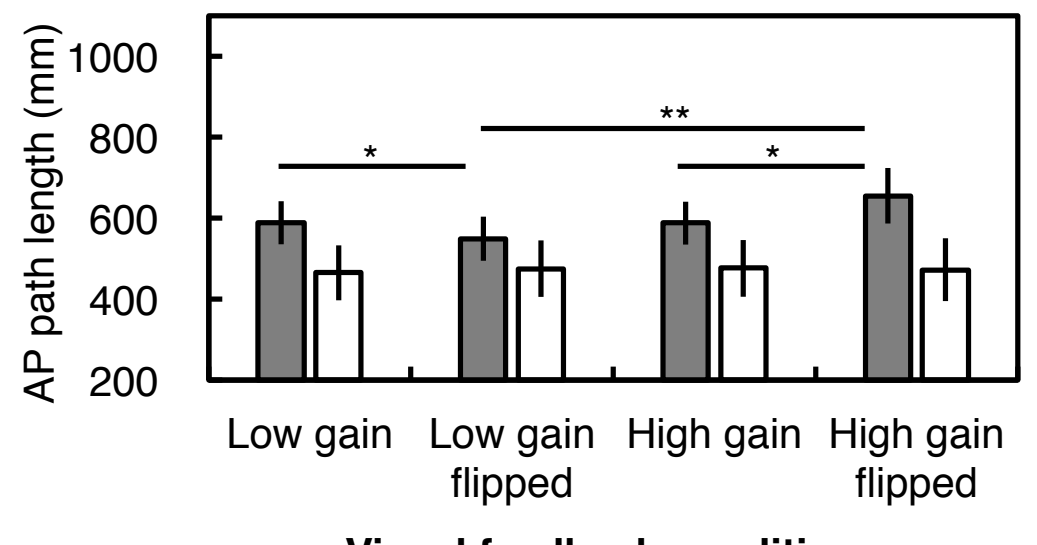

B

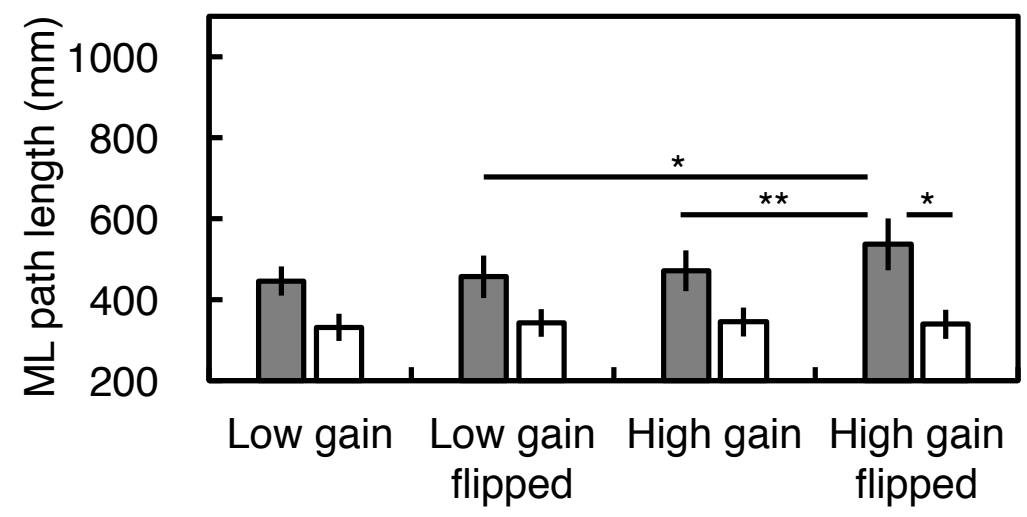

D

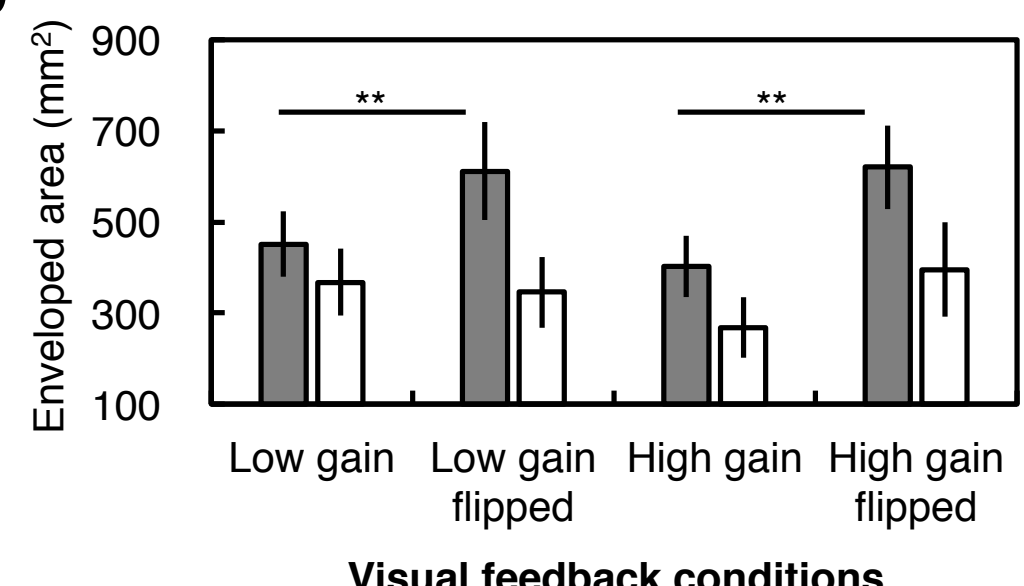

$\square$ Voluntary-control group $\quad \square$ Observation group 


\section{Table $\mathbf{1}$ (on next page)}

Characteristics of participants.

Mean value is followed by standard deviation in parentheses. Asterisk indicates Welch's correction for violation of the homogeneity assumption. 


\begin{tabular}{|c|c|c|c|}
\hline & $\begin{array}{l}\text { Voluntary- } \\
\text { control group }\end{array}$ & $\begin{array}{l}\text { Observation } \\
\text { group }\end{array}$ & Statistics for group differences \\
\hline Sex & $\begin{array}{r}\text { Male } 7, \\
\text { female } 3\end{array}$ & $\begin{array}{r}\text { Male 5, } \\
\text { female } 5\end{array}$ & $\chi^{2}(1)=0.83, p=.361, \varphi=.204$ \\
\hline Age (year) & $19.40(1.43)$ & $18.80(0.63)$ & $\begin{array}{r}t\left(12.39^{*}\right)=1.21, p=.248, d= \\
.543\end{array}$ \\
\hline Height (m) & $1.680(0.091)$ & $1.671(0.066)$ & $t(18)=0.25, p=.803, d=.114$ \\
\hline Weight (kg) & $55.20(6.30)$ & $56.40(7.29)$ & $t(18)=0.39, p=.698, d=.177$ \\
\hline Body mass index $\left(\mathrm{kg} / \mathrm{m}^{2}\right)$ & $19.54(1.41)$ & $20.12(1.41)$ & $t(18)=0.92, p=.369, d=.412$ \\
\hline
\end{tabular}




\section{Table 2 (on next page)}

Summary of the main effects and interactions of three factors on each dependent variable.

Degrees of freedom were 1 and 18. Statistically significant values $(p<.05)$ are bolded. 


\begin{tabular}{|c|c|c|c|c|c|c|c|c|}
\hline & & Instruction & Gain & Flip & $\begin{array}{l}\text { Instruction } \\
\times \text { Gain } \\
\end{array}$ & $\begin{array}{l}\text { Instruction } \\
\times \text { Flip }\end{array}$ & $\begin{array}{l}\text { Gain } \\
\times \text { Flip } \\
\end{array}$ & $\begin{array}{l}\text { Instruction } \times \\
\text { Gain } \times \text { Flip }\end{array}$ \\
\hline \multirow{3}{*}{$\begin{array}{l}\text { Sense of } \\
\text { control }\end{array}$} & $F$ & 16.46 & 2.06 & 0.06 & $<0.01$ & 0.14 & 0.37 & 2.95 \\
\hline & $p$ & .001 & .169 & .804 & .972 & .710 & .552 & .103 \\
\hline & $\eta_{\mathrm{G}}^{2}$ & .355 & .036 & $<.001$ & $<.001$ & $<.001$ & .001 & .007 \\
\hline \multirow{3}{*}{$\begin{array}{l}\text { Total path } \\
\text { length }\end{array}$} & $F$ & 3.29 & 7.50 & 2.39 & 5.71 & 1.42 & 6.09 & 11.02 \\
\hline & $p$ & .086 & .014 & .139 & .028 & .249 & .024 & .004 \\
\hline & $\eta_{\mathrm{G}}^{2}$ & .149 & .007 & .002 & .005 & .001 & .003 & .006 \\
\hline \multirow{3}{*}{$\begin{array}{l}\text { ML path } \\
\text { length }\end{array}$} & $F$ & 5.39 & 4.24 & 3.76 & 2.92 & 2.86 & 2.22 & 7.65 \\
\hline & $p$ & .032 & .054 & .068 & .104 & .108 & .153 & .013 \\
\hline & $\eta_{\mathrm{G}}^{2}$ & .214 & .012 & .006 & .008 & .004 & .001 & .005 \\
\hline \multirow{3}{*}{ AP path length } & $F$ & 1.85 & 12.47 & 0.79 & 9.05 & 0.28 & 7.01 & 11.62 \\
\hline & $p$ & .191 & .002 & .387 & .008 & .605 & .016 & .003 \\
\hline & $\eta_{\mathrm{G}}^{2}$ & .091 & .005 & .001 & .004 & $<.001$ & .004 & .006 \\
\hline \multirow{3}{*}{$\begin{array}{l}\text { Enveloped } \\
\text { area }\end{array}$} & $F$ & 2.93 & 0.34 & 12.04 & $<0.01$ & 3.86 & 4.09 & 0.82 \\
\hline & $p$ & .104 & .565 & .003 & .959 & .065 & .058 & .377 \\
\hline & $\eta_{\mathrm{G}}^{2}$ & .110 & .002 & .054 & $<.001$ & .018 & .011 & .002 \\
\hline
\end{tabular}

ML: medio-lateral; AP: antero-posterior. 


\section{Table 3(on next page)}

Correlations among subjective and postural indices from all participants under each visual feedback condition.

Values are Pearson's correlation coefficients $(r)$ followed by $p$ values (two-tailed; false discovery rate corrected) in parentheses. Degrees of freedom were 78. Statistically significant values $(p<.05)$ are bolded. 


\begin{tabular}{|c|c|c|c|c|}
\hline & Sense of control & Total path length & ML path length & AP path length \\
\hline Total path length & .408 (.001) & & & \\
\hline ML path length & $.492(.001)$ & $.900(.001)$ & & \\
\hline AP path length & .301 (.012) & $.951(.001)$ & $.723(.001)$ & \\
\hline Enveloped area & $.151(.226)$ & $.125(.298)$ & $.208(.091)$ & $.050(.656)$ \\
\hline
\end{tabular}

ML: medio-lateral; AP: antero-posterior. 